\title{
EFFECT OF HIGH-VELOCITY LOW-AMPLITUDE THRUST MANIPULATION OF UPPER CERVICAL SPINE ON PULMONARY FUNCTION TEST AND HEADACHE DISABILITY INDEX IN PATIENTS WITH MIGRAINE HEADACHE
}

\author{
SONU PUNIA, MANOJ MALIK, SHALU JANGRA, JASPREET KAUR, VARUN SINGH \\ Guru Jambheshwar University of Science and Technology, Department of Physiotherapy, Hisar, India
}

Mailing address: Shalu Jangra,Guru Jambheshwar University of Science and Technology, Department of Physiotherapy, Hisar-125001, tel.: +09466373990, e-mail: shalujangra@gmail.com

\begin{abstract}
Introduction. Migraine is a common debilitating disorder of neurovascular origin which affects younger adults, especially women. Material and Methods. It was an experimental study design. A convenience type of sampling was used for recruitment of the subjects. A total of 21 participants diagnosed with migraine ( 6 males and 15 females) fulfilling the inclusion criteria were taken for this study and assigned to one of the two groups (experimental or control group). PFT measured with PFT apparatus (Spirolab 111, Serial No. A23-053 13974) and HDI score were calculated before the start of the intervention and after the intervention. Afterwards, PFT was performed by each participant followed by HVLA thrust manipulations to the migraine patients and an equal period of rest to the control group. Results. There are no significant differences between baseline and post-intervention outcome variables and in mean changes between the two groups. One-way ANOVA was used to analyze the change in HDI score which was calculated three times (pre- prior to intervention, post- after the intervention and 1 month after the intervention). The experimental group showed a significant reduction in the score after the manipulation. It was found that FVC and PEF were positively correlated to FEVI, and FVC was also positively correlated to PEF. Conclusions. The findings of the study revealed that high-velocity thrust manipulations of upper cervical spine were not effective in improving pulmonary functions of migraine patients although it was found effective in improving subjective symptoms of the patients evaluated through HDI.
\end{abstract}

Key words: migraine, manipulation, mobilization, pulmonary function test

\section{Introduction}

Migraine is a common debilitating disorder of neurovascular origin which influences younger population, especially females. Diagnosis of migraine is mainly based on the clinical presentation of the patient, but the non-invasive neuroimaging tools exclude some other causes of headache [1]. The incidence rises in younger adults (15-24 years of age), the prevalence is greatest in middle decades ( $35-45$ years). The rate of prevalence of migraine is found to be $17.6 \%$ in female adults and $5.7 \%$ in the male population in the United States. The cumulative lifetime incidence of migraine is $43 \%$ in women and $18 \%$ in men [1]. One study concluded that $25 \%$ of headache patients met the set criteria for migraine even when they were not diagnosed as migraineurs, while another study reported that $50 \%$ of headache patients were undiagnosed with migraine and even when migraine was confirmed, it was often undertreated $[1,2]$.

Migraine results from disrupted modulation of the trigeminovascular system and activation of the system [3]. Pathophysiology of migraine headache involved both diencephalic and brainstem nuclei. Neuro-imaging techniques revealed the activation of dorsal pons and the premonitory state of hypothalamus [3]. Patients with migraine experienced aura symptoms like yawning (activation of the dopaminergic system due to the involvement of hypothalamic and ventral tegmental areas), feeling of thirst (may be caused by reduced vasopressin), neck stiffness or polyuria prior (up to three days) to the headache and mood changes (hypothalamic connections with the limbic system) [3].

A unilateral type of migraine headache occurs in $60 \%$ and bilateral in $40 \%$ of cases. Migraineurs report 15\% "side-locked" headaches on the ipsilateral side. The pain is often more intense in the fronto-temporal and orbital area than when it involved parietal and occipital areas. It also involved any region of the head or face (parietal region, the upper or lower jaw or teeth, the malar eminence, and the upper anterior neck) [1].

The nature of pain is throbbing in $85 \%$ of migraine patients, although up to $50 \%$ described it as non-throbbing pain. Up to $75 \%$ of migraineurs reported head pain, unilateral or bilateral tightness, stiffness or throbbing pain in the posterior region of the head. Migraine which persists for more than 3 days (72 hours) is termed as status migrainosus. Intensity of pain is moderate to severe in $80 \%$ of patients and mild in $20 \%$ of patients. The pain, which is usually increased by physical exertion, is associated with nausea (80\%), vomiting (30\%) and photophobia (90\%) [2]. Women experienced migraine attacks usually before or during menses cycles [1].

Previous research revealed that chronic neck pain patients did not have ideal pulmonary function test (PFT) values. Intensity of pain and kinesiophobia is associated with cervical spinal muscle dysfunction along with respiratory dysfunction [4]. Ozge et al. (2006) carried out a study on the relationship between migraine and atopic disorders and found that PFT screening showed general decreased pulmonary capacity in patients of atopic disorders [5].

Research shows that spinal manipulation technique (SMT) is an effective intervention for a certain type of headache but it is uncertain how much this intervention affects the intensity of headache and produces any long-term changes in these conditions [6]. Some patients with migraine headache do not tolerate medical treatment because of associated pathological disorders. SMT may be considered in these situations where other 
medical treatments are ineffective or poorly tolerated [7]. However, long-term effects of manipulation are still uncertain. There is a lack of research on the effectiveness of SMT, which appears to have more impact on cervicogenic headache, tension-type headache and migraine headache than massage [8]. Asthmatic and allergic patients have a higher frequency of migraine-type headache and low respiratory functional capacities $[9,10,11]$. Migraine is the most important diagnosis in this population; sleep and breathing disorders have long been recognized as a cause of headache in this population [12].

Decreased trunk flexibility and decreased diameters of thoracic cavity can lead to reduced respiratory function value. Thoracic mobilization exercises can increase trunk flexibility. Thus, mobilization of thorax and cervical vertebrae may increase trunk flexibility and enhance respiratory functional capacities [13]. Therefore, the aim of the study was to evaluate the effect of high-velocity low-amplitude thrust manipulation (HVLA) of upper cervical spine on lung capacities and headache symptoms in patients with migraine headache.

\section{Material and Methods}

It was an experimental study design. A convenience type of sampling was used for recruitment of the subjects. A total of 21 participants diagnosed with migraine (6 males and 15 females) fulfilling the inclusion criteria were taken for this study and assigned to one of the two groups (experimental or control group). Patients were recruited at Jindal Institute of Medical Sciences, Hisar, Haryana, India. The inclusion criteria for the study were as follows: participants diagnosed with migraine, aged between 20 to 50 years, headache disability index (HDI) higher than $30 \%$. Patients with any musculoskeletal, neurological or cardio-respiratory disorders which could affect the study variable were excluded.

Patients suffering from migraine diagnosed by a neurosurgeon were assessed for inclusion and exclusion criteria. Those who fulfilled the inclusion and exclusion criteria were told about the study in their mother tongue. Then, the willing participants were asked to give a written informed consent and were included into the experimental and control group. Afterwards, PFT was performed by each participant followed by HVLA thrust manipulations to the migraine patients and an equal period of rest to the control group. PFT measured with PFT apparatus (Spirolab 111, Serial No. A23-053 13974) and HDI score were calculated before the start of the intervention and after the intervention.

PFT was performed as per the guidelines of the American Thoracic Society. PFT was performed by the trained technician of the Jindal Hospital. The subjects were asked to sit on the stool in a correct posture with the head slightly elevated. They were informed about the procedure of PFT, and the demonstration of the performance of PFT procedure was given to each participant. Then, the patients were asked to inhale rapidly and completely. After that, they were asked to exhale forcefully and completely in a mouthpiece with a clipped nose. This test was repeated for a minimum of three trials and then the final reading of all parameters of PFT was taken and recorded on the data collection form. The parameters of PFT are Forced Vital Capacity (FVC), Forced Expiratory Volume (FEV1), FVC/FEVl and Peak Expiratory Flow (PEF).

The patient was in a supine position; the head (off the couch) was held by the therapist standing on the head side of the couch facing the patient's feet. High-velocity low-amplitude (HVLA) cervical manipulation technique was applied to patients in the experimental group. HDI score was calculated for all the migraine patients following PFT and one month after HVLA cervical manipulations.

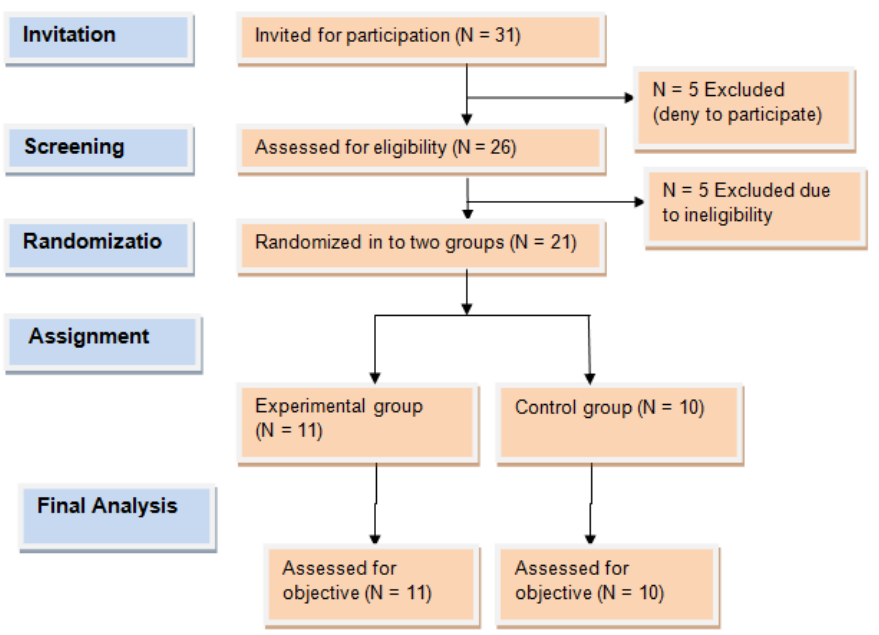

Figure 1. Flow chart of selection of participants.

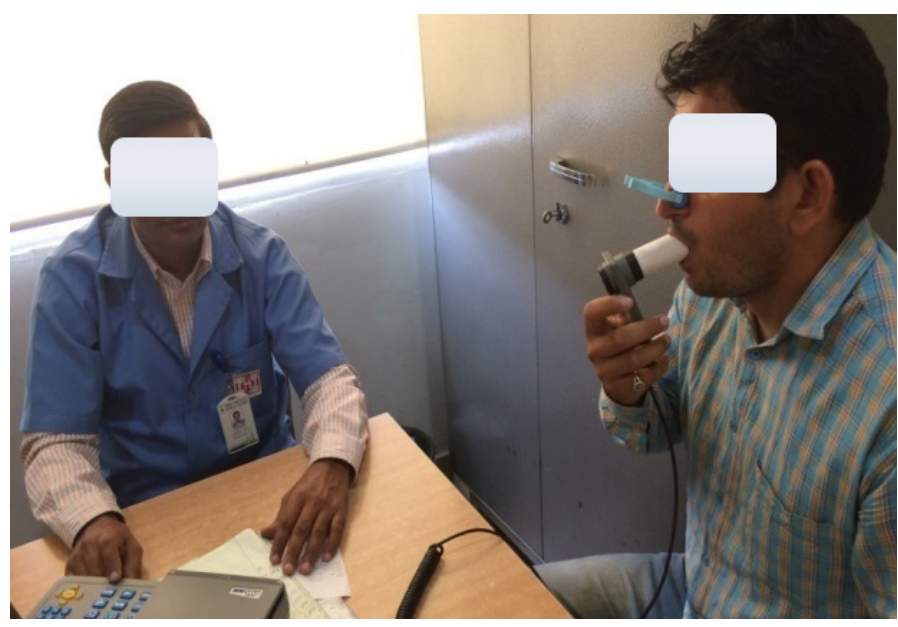

Figure 2. Patient performing PFT.

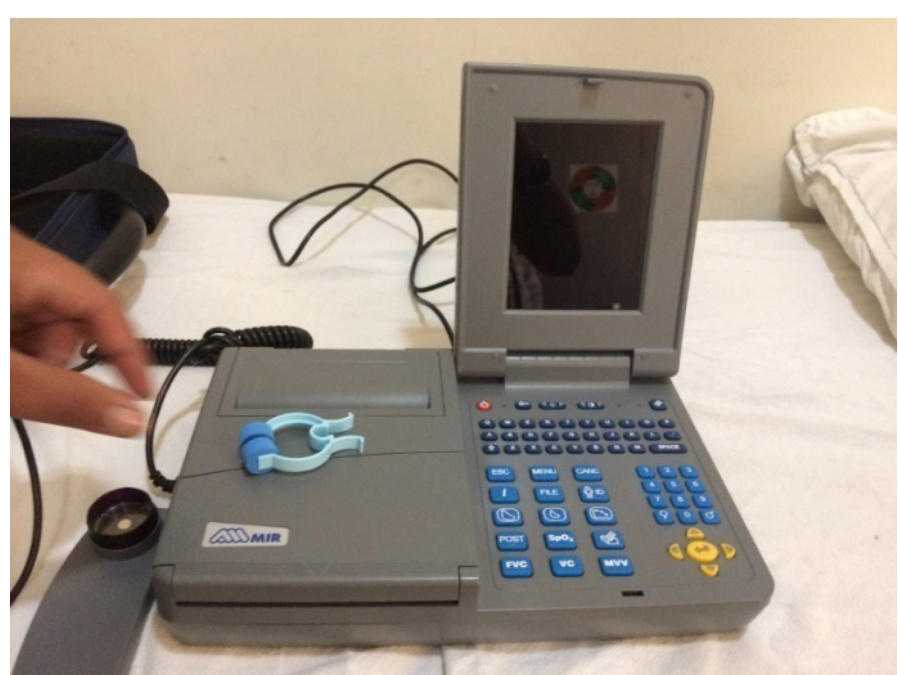

Figure 3. PFT apparatus. 


\section{Data analysis}

The data was analyzed using SPSS version 21. Significance level was set at 0.05. Chi-square test and t-test were used for the comparison of categorical and continuous variables, respectively. One-way ANOVA test was used for the comparison of repeated measures and correlation was analyzed using Pearson correlation test.

\section{Results}

A total of 21 participants were recruited for this study. Eleven patients were assigned to the experimental group, while 10 patients (age matched) were assigned to the control group. Table 1 compares demographic characteristics of the study population. As seen from the table, there are no differences in age, weight, height and BMI between the groups. The significance level was set at 0.05 .

There are no significant differences between baseline and post-intervention outcome variables between the two groups (Tab. 2).

There are no significant differences in mean changes between baseline and post-intervention outcome variables between the two groups (Tab. 3).

In table 4, one-way ANOVA was used to analyze the change in HDI score which was calculated three times (pre- prior to intervention, post- after the intervention and 1 month after the intervention) in the experimental group. It showed a significant reduction in the score after the manipulation.

In Table 5, Spearman correlation was applied to analyze correlations between the variables used in this study. It was found that FVC and PEF were positively correlated to FEVl, and FVC was also positively correlated to PEF.

\section{Discussion}

The purpose of the present study was to evaluate the effect of HVLA upper cervical manipulation on PFT and HDI in patients with migraine. Previous studies revealed that the intensity of headache can be increased by a noxiously stimulating receptor of tissues, joint capsules, ligaments and paraspinal muscles (innervated by the cervical nerve roots $\mathrm{Cl}-\mathrm{C} 3$ ) [8]. So, upper cervical manipulation may stimulate the phrenic nerve having root value C3-C5 which supplies the diaphragm which is the main muscle of inspiration. Patients with chronic neck pain do not have ideal pulmonary function capacity, i.e. reduced FVC, expiratory reserve volume and maximum voluntary ventilation [4].

There were no significant mean differences between the groups in terms of age, height, weight and BMI. So, both groups were homogeneous over these parameters which are important factors to affect pulmonary function. Hence, in the present study, pulmonary function between the two groups was not affected by demographic variables like age, height, weight and BMI. This gives strength to the present study.

Bhatti et al. found variations in pulmonary vital capacity of people in relation to their demographic variables (height within the same ethnic and age groups) [14]. Pruthi \& Multani reported that the values of all lung function tests, namely FVC, FEVl, PEF, FEVI/FVC, SVC and MVV were found to be negatively correlated with age. It was concluded that the lung functions significantly declined with age [15]. Chen et al. reported that an increase in weight was directly related to pulmonary dysfunction and the impact of increased weight was larger in males as compared to females [16].
Table 1. Demographic data of study population

\begin{tabular}{|l|c|c|c|c|}
\hline Variables & $\begin{array}{c}\text { Experimental } \\
\text { group }\end{array}$ & Control group & t-test & $p$-value \\
\hline Age & $35.90 \pm 8.837$ & $35.45 \pm 8.442$ & $0.12^{\mathrm{NS}}$ & 0.91 \\
\hline Weight & $65.50 \pm 14.804$ & $62.00 \pm 11.559$ & $0.61^{\mathrm{NS}}$ & 0.55 \\
\hline Height & $1.622 \pm 0.083$ & $1.631 \pm 0.075$ & $-0.28^{\mathrm{NS}}$ & 0.78 \\
\hline $\mathrm{BMl}$ & $24.651 \pm 3.626$ & $23.239 \pm 4.000$ & $0.84^{\mathrm{NS}}$ & 0.41 \\
\hline Gender $(\mathrm{M}: \mathrm{F})$ & $3: 8$ & $3: 7$ & $0.02^{\mathrm{NS}}$ & 0.89 \\
\hline
\end{tabular}

* - Data is in form MEAN \pm SD; NS - Non-significant.

Table 2. Comparison of means of baseline and post-intervention outcome measure between two groups

\begin{tabular}{|l|c|c|c|c|}
\hline \multicolumn{1}{|c|}{ Variables } & $\begin{array}{c}\text { Experimental } \\
\text { group }\end{array}$ & $\begin{array}{c}\text { Control } \\
\text { group }\end{array}$ & t-test & p-value \\
\hline Baseline FVC & $3.02 \pm 0.588$ & $3.10 \pm 0.854$ & $-0.26^{\mathrm{NS}}$ & 0.80 \\
\hline Post FVC & $2.99 \pm 0.546$ & $3.12 \pm 0.879$ & $-0.424^{\mathrm{NS}}$ & 0.676 \\
\hline Baseline FEV1 & $2.56 \pm 0.501$ & $2.62 \pm 0.716$ & $-0.22^{\mathrm{NS}}$ & 0.83 \\
\hline Post FEV1 & $2.52 \pm 0.441$ & $2.64 \pm 0.713$ & $-0.464^{\mathrm{NS}}$ & 0.648 \\
\hline $\begin{array}{l}\text { Baseline FEV1/ } \\
\text { FVC }\end{array}$ & $85.09 \pm 4.92$ & $84.57 \pm 4.96$ & $0.24^{\mathrm{NS}}$ & 0.18 \\
\hline Post FEV1/FVC & $84.90 \pm 5.94$ & $84.91 \pm 4.986$ & $-.254^{\mathrm{NS}}$ & 0.803 \\
\hline Baseline PEF & $6.52 \pm 1.92$ & $6.35 \pm 2.01$ & $0.19^{\mathrm{NS}}$ & 0.85 \\
\hline Post PEF & $6.65 \pm 1.83$ & $5.98 \pm 2.095$ & $0.768^{\mathrm{NS}}$ & 0.455 \\
\hline
\end{tabular}

* - Data is in form MEAN \pm SD; NS - Non-significant.

Table 3. Comparison of mean change in values of FVC, FEVl, FEVI/ FVC, PEF between two groups

\begin{tabular}{|l|c|c|c|c|}
\hline \multicolumn{1}{|c|}{ Variables } & $\begin{array}{c}\text { Experimental } \\
\text { group }\end{array}$ & Control group & t-test & $p$-value \\
\hline Post FVC & $0.300 \pm 0.227$ & $-0.021 \pm 0.170$ & $0.576^{\mathrm{NS}}$ & 0.572 \\
\hline Post FEV1 & $0.0373 \pm 0.201$ & $-0.022 \pm 0.159$ & $0.743^{\mathrm{NS}}$ & 0.467 \\
\hline Post FEV1/FVC & $0.181 \pm 3.309$ & $-0.340 \pm 4.734$ & $0.295^{\mathrm{NS}}$ & 0.771 \\
\hline Post PEF & $-0.122 \pm 0.565$ & $0.369 \pm 1.572$ & $-0.972^{\mathrm{NS}}$ & 0.343 \\
\hline
\end{tabular}

* - Data is in form MEAN \pm SD; NS - Non-significant.

Table 4. Headache Disability Index (HDI) Score variability in experimental group

\begin{tabular}{|l|c|c|c|}
\hline \multicolumn{1}{|c|}{ Variables } & Mean \pm SD & F & p-value \\
\cline { 1 - 2 } HDI-pre & $44.91 \pm 11.64$ & & \multirow{2}{*}{$3.79^{*}$} \\
\cline { 1 - 2 } HDI-post & $34.00 \pm 7.85$ & .034 \\
\cline { 1 - 2 } HDI-post 1 Month & $38.36 \pm 8.09$ & & \\
\hline
\end{tabular}

* - Data is in form MEAN $\pm \mathrm{SD}$; * - Significant at p-value less than 0.05; HDI - Headache Disability Index.

Table 5. Correlation Table

\begin{tabular}{|l|c|c|}
\hline \multicolumn{1}{|c|}{ Variables } & r-value & p-value \\
\hline FVC-FEV1 & $.954^{*}$ & 0.001 \\
\hline PEF-FEV1 & $.898^{*}$ & 0.001 \\
\hline FVC-PEF & $.889^{*}$ & 0.001 \\
\hline
\end{tabular}

* - Significant at p-value less than 0.05 . 
The male percentage in the present study was lower in both groups, i.e. $27.3 \%$ in the experimental group and 30\% in the control group compared to females $(72.3 \%$ in the experimental group and $70 \%$ in the control group). This might have been caused by greater prevalence of migraine among females [1]. Furthermore, chronic smoking was the exclusion criterion for the study, so most of the male patients were excluded because of smoking history. Hedenstrom et al. found that heavy tobacco smoking caused pulmonary dysfunction in younger adults to elderly people (20-70 years) [16].

The means of baseline measurements of outcome variables in both groups were homogeneous. So, there were no differences in baseline reading of all the participants included in the study and it did not affect the post-intervention readings in the present study.

In the current study, the comparison of mean changes in values of FVC, FEV1, FEV1/FVC and PEF between the two groups did not show any significant changes. Therefore, it can be stated that there is no effect of upper cervical HVLM manipulation on PFT parameters. Wall et al. also did not support the use of manual therapy to provide a short-term benefit in respiratory function to healthy adults [17]. In contrast, Kessinger et al. reported that pulmonary function improved significantly in subjects under upper cervical chiropractic care because the sample size was large and all the subjects were of vertebral subluxation (not of migraine) and the treatment sessions were multiple [18].

Masarsky et al. also found a significant improvement in both FVC and FEVl after four to six adjustments to the spine [19]. The non-significant results in the present study might be due to a single session of treatment given to patients. Additionally, a strong correlation was found between FEVI and PEF in the present study, which is in agreement with previous studies $[20,21]$.

The HDI score in this study showed a significant improvement in symptoms of patients specifically in the intensity of headache. It indicates that HVLT manipulation is an effective treatment procedure. It was also suggested by Chaibi et al. that massage therapy, physiotherapy, relaxation and chiropractic spinal manipulative therapy might be as efficient as propranolol and topiramate in the prophylactic management of migraine [22]. Some manipulative therapists suggest that migraine patients with cervical spondylosis are most likely to benefit from manipulation as periarticular adhesions are broken down. Cyriax suggests that manipulation might reduce pressure from exfoliated articular cartilage on the vertebral artery and the sympathetic fibres running with it [23]. El-Sodany et al. also reported that SNAGs mobilization and manipulation were found to be effective treatments more than exercises alone in the treatment of cervical spine disorders [24].

A small sample size and short duration of the study are the biggest limitations of the present study. Secondly, a single treatment session is also a limitation, as other studies in which multiple manipulation sessions were used revealed the effectiveness of manipulation on PFT [18].

The same study can be carried out with a larger sample size and multiple sessions of HVLT manipulations can be applied to see the effect on PFT. Manipulation in combination with mobilization can be used and analyzed to see the effect on PFT and subjective relief of migraine symptoms.

\section{Conclusion}

The findings of the study revealed that high-velocity thrust manipulations of upper cervical spine were not effective in im- proving pulmonary functions of migraine patients although it was found effective in improving subjective symptoms of the patients evaluated through HDI.

\section{Acknowledgments}

The authors thank the study participants as well as institutions that helped to prepare the manuscript and provided technical assistance.

\section{References}

1. Bartleson J.D., Cutrer F.M. (2010). Migraine update. Diagnosis and treatment. Minnesota Medicine 93(5), 36-41. PMID: 20572569.

2. Evans R.W. (2014). The clinical features of migraine with and without aura. Practical Neurology 13, 26-32.

3. Holland P.R., Afridi S. (2014). Migraine pathophysiology. Advances in Clinical Neuroscience E Rehabilitation 13(7), $19-21$.

4. Dimitriadis Z., Kapreli E., Strimpakos N., Oldham J. (2014). Pulmonary function of patients with chronic neck pain: a spirometry study. Respiratory Care 59(4), 543-549. DOI: $10.4187 /$ respcare.01828

5. Özge A., Özge C., Öztürk C., Kaleagasi H., Özcan M. et al. (2006). The relationship between migraine and atopic disorders - the contribution of pulmonary function tests and immunological screening. Cephalalgia 26(2), 172-179. DOI: 10.1111/j.1468-2982.2005.01021.x

6. Astin J.A., Ernst E. (2002). The effectiveness of spinal manipulation for the treatment of headache disorders: a systematic review of randomized clinical trials. Cephalalgia 22(8), 617-623. DOI: 10.1046/j.1468-2982.2002.00423.x

7. Chaibi A., Benth J.Š.,Tuchin P.J., Russell M.B. (2017). Chiropractic spinal manipulative therapy for migraine: a threearmed, single-blinded, placebo, randomized controlled trial. European Journal of Neurology 24(1), 143-153. DOI: 10.1111/ene.13166

8. Bronfort G., Assendelft W.J., Evans R., Haas M., Bouter L. (2001). Efficacy of spinal manipulation for chronic headache: a systematic review. Journal of Manipulative and Physiological Therapeutics 24(7), 457-466. PMID: 11562654.

9. Ingraham P. (2016). The Respiration Connection. 'How dysfunctional breathing might be a root cause of a variety of common upper body pain problems and injuries'. Retrived January 20, 2021, from https://www.painscience.com/articles/respiration-connection.php

10. Ghanbari A., Ghaffarinejad F., Mohammadi F., Khorrami M., Sobhani S. (2008). Effect of forward shoulder posture on pulmonary capacities of women. British journal of sports medicine 42(7), 622-623. DOI: 10.1136/bjsm.2007.040915

11. Gungen A.C., Gungen B. (2017). Assessment of headache in asthma patients. Pakistan Journal of Medical Sciences 33(1), 156-161. DOI: 10.12669/pjms.331.11720

12. Peres M.F., Lerário D.D., Garrido A.B., Zukerman E. (2005). Primary headaches in obese patients. Arquivos de Neuro-Psiquiatria 63(4), 931-933. PMID: 16400407. DOI:10.1590/ s0004-282x2005000600005

13. Jang S.H., Bang H.S. (2016). Effect of thoracic and cervical joint mobilization on pulmonary function in stroke patients. Journal of Physical Therapy Science 28(1), 257-260. DOI: $10.1589 /$ jpts.28.257

14. Bhatti U., Rani K., Memon M.Q. (2014). Variation in lung volumes and capacities among young males in relation to 
height. Journal of Ayub Medical College Abbottabad 26(2), 200-202.

15. Pruthi N., Multani N.K. (2012). Influence of age on lung function tests. Journal of Exercise Science and Physiotherapy $8(1), 1-6$.

16. Chen Y., Horne S.L., Dosman J.A. (1993). Body weight and weight gain related to pulmonary function decline in adults: a six year follow up study. Thorax 48(4), 375-380. DOI: $10.1136 /$ thx.48.4.375

17. Wall B.A., Peiffer J.J., Losco B., Hebert J.J. (2016). The effect of manual therapy on pulmonary function in healthy adults. Scientific Reports 6(1), 1-4. DOI: 10.1038/srep33244

18. Kessinger R. (1997). Changes in pulmonary function associated with upper cervical specific chiropractic care. Journal of Vertebral Subluxation Research 1(3), 43-50.

19. Masarsky C.S., Weber M. (1988). Chiropractic management of chronic obstructive pulmonary disease. Journal of Manipulative and Physiological Therapeutics 11(6), 505-510.

20. Lebowitz M.D., Knudson R.J., Robertson G., Burrows B. (1982). Significance of intraindividual changes in maximum expiratory flow volume and peak expiratory flow measurements. Chest 81(5), 566-570. DOI: 10.1378/chest.81.5.566

21. Ritchie B. (1962). A comparison of forced expiratory volume and peak flow in clinical practice. The Lancet 280(7250), 271-273. DOI: 10.1016/S0140-6736(62)90174-5

22. Chaibi A., Tuchin P.J., Russell M.B. (2011). Manual therapies for migraine: a systematic review. The Journal of Headache and Pain 12(2), 127-133. DOI: 10.1007/s10194-011-0296-6

23. Parker G.B., Tupling H., Pryor D.S. (1978). A controlled trial of cervical manipulation for migraine. Australian and New Zealand Journal of Medicine 8(6), 589-593. DOI: 10.1111/ j.1445-5994.1978.tb04845.x

24. El-Sodany A.M., Alayat M.S.M., Zafer A.M.I. (2014). Sustained natural apophyseal glides mobilization versus manipulation in the treatment of cervical spine disorders: a randomized controlled trial. International Journal of Advanced Research 2(6), 274-280.

Submitted: February 09, 2021

Accepted: February 17, 2021 